\title{
Section on Ophthalmology College of Physicians of Philadelphia
}

Meeting 17. Oktober 1899

Risley, S. D., berichtet über einen Fall von Glaucom bei einer 7 7jährigen Patientin. Das

Glaucom trat 3 Jahre nach einer erfolgreich vorgenommenen einfachen Extraction auf. Als

Ursache bezeiclmet R. eine 5 Wochen vorher im Verlaufe einer Influenza aufgetretene Iritis, die zu r1ºgförmigen hinteren Synechíen geführt hatte. Eine Iridectomie beseitigte das Glaucom.

Sweet, W. Al., zeigte einen Patienten mit doppelseitiger Seli-nei'venatrofie infolge

Darmblutung. 6 Tage nach der Darmblutung kam es zur Blindheit auf dem líuken Auge. während auf dem rechten Auge

Therapoutische TJmsch.au. $\quad 27 /$

noch eine kleine Zone temporal vom Fixationspunkt erhalten blieb. Ophthalmoskopisch war eine Verschmälerung der Netzhautarterien, Blässe der Papillen mit leieht verwischten Rändern und leichtes Netzhautödem besonders in der Fovea-Gegend nachweisbar. Auf dem rechten Auge lief vom unteren Rande der Papille eine Ciliararterie gegen die Fovea, wodurch sicli die Intactlieit der kleineu Zone erklärt.

Hansell, 11. F spricht über die physiologischen Variationen in der Grösse des blinden Fleckes. (Siehe Referat in dem aussteheuden $\mathrm{S}^{1}$ /8inestralbericht fur die 2. Hälfte des Jahres 189!).)

1) e n i g-New York, Therapeutische Umschau.

Untersuchungen über die Einwirkung neuerer Antiseptica auf inñcierte Hornhautwunden. Dr. Hauenschild - Würzburg. (Münchener med. $\Lambda$ Vochenschr. No. 5. 1900.)

Verf. hat zur Klärung der noch sehr geteilten Meinungen über den Wert der Antiseptica am Auge überhaupt und der zweckmässigsten Art ihrer Anwendung, unter Anschluss an die frühersn Untersuchungen von Eversbusch und Bach einige neue Versuche an künstlich in-ficierten Wunden geniacht.

Bekanntlich kam Bach zu folgenden Resultaten: Er setzte auf der Hornhaut von Kaninchen Verletzungen durch in †icierte Lanzen (Staphylococcus pyogenes) und berieselte die Wunden gleich darauf mit antiseptischen Lösungen. Unter 14 Versuclien mit Bespülen von Sublimat 1/1000 gelang es, wie die bakteriologische Untersuchung erwies. nur einmal, sämtlíche Staphylokokken abzutöten. Als keimtötend erwiesen sich nur 1-2 pCt. Hydrargyrum oxycyanatum, 1 proc. .lod-trichlorid und 1-2proc. Pyoktanin.

Hauenschild verfuhr ähnlich wie Bach. Er setzte Hornhaut-verletzungen mit durch Micrococcus pyogenes inficierten Lanzen, berieselte dann sofort die verletzten SteUen (Taschen in der Hornhaut) mit antiseptischen Flüssigkeiten und einer Undine, trug darauf die Hornhaut, welche vorsichtshalber zur Entfernung der oberflächlich an-haftenden Keime noch in Kochsalz abgespült wurde, ab und verimpfte sie auf Petri'sche Schälchen mit geeigneten Nährböden. Es $\lambda$ vurden 10 Versuche mit I pCt., $5 \mathrm{j}>\mathrm{Ct}$. und $10 \mathrm{pCt}$. Pro-targol, 4 Versuche mit $1 \mathrm{pCt}$. und 2 $\mathrm{j}>\mathrm{Ct}$. Argentum nitricum und 14 Versuche mit Hydrarg. oxycyanatum in Lösungen yon 1 : 5000 bis $1: 100$ angestellt. 
In alien Fallen zeígten sich die Nährböden mit zahlreichen Kolonien des Eítererregers übersäet. D. Dasselbe günstige Ergebnis zeigte sich sogar noch bei Ver-wendung von 1-2 pCt. Hydrarg. oxycyanat.

Dieser auffallende Unterschied gegen die früheren Resultate Bach's erklärte sich später durch folgenden Umstand: 